\title{
Surgical techniques for total sacrectomy and spinopelvic reconstruction
}

\author{
Ho-Yeol Zhang, M.D., Ph.D., Issada Thongtrangan, M.D., \\ Raju S. V. Balabhadra, M.D., Judith A. Murovic, M.D., and Daniel H. Kim, M.D. \\ Department of Neurosurgery, Stanford University Medical Center, Stanford, California; and \\ Department of Neurosurgery, National Health Insurance Corporation Ilsan Hospital, Koyang, Korea
}

\begin{abstract}
The surgical management of sacral tumors requires partial or total sacrectomy and spinopelvic reconstruction. These lesions present a great surgical challenge, because most spine surgeons are unfamiliar with the techniques required for these procedures. The authors describe a step-by-step operative technique and provide several illustrations.

Total sacrectomy is performed by sequential anterior and posterior approaches that involve a rectus abdominis pullthrough pedicle flap reconstruction. The anterior procedure is an intraperitoneal approach used to expose the anterior aspect of the tumor, to ligate the main tumor vessels, and to conduct an anterior partial sacrectomy. After this, the rectus abdominis myocutaneous flap, based on the inferior epigastric vessel, is prepared, and a posterior sacrectomy is performed, dividing all sacral nerve roots in the thecal sac. After complete en bloc extirpation of the sacrum with tumor, spinopelvic reconstruction and closure with a myocutaneous flap are performed. Spinopelvic reconstruction is undertaken using a modified Galveston technique or double iliac screw fixation combined with posterior lumbar segmental fixation. These provide a long lever arm within the ilium to counteract the forces exerted by the lumbar spine.

Understanding the nature of the disease as well as the biomechanics of the lumbosacral pelvic area and spinopelvic fixation will help surgeons select the appropriate treatment for sacral tumors.
\end{abstract}

\section{KEY WORDS • sacrum • spinal tumor • total sacrectomy - spinopelvic reconstruction}

Tumors of the sacrum frequently pose a complex problem for surgical management and stabilization because of the anatomical and biomechanical factors of this transition zone. Although some benign tumors such as osteoblastoma, sacral cyst, and osteochondroma can be easily excised, removal of aggressive tumors including GCT and aneurysmal bone cyst is difficult because of their size, vascularity, and extension. The common primary malignant tumors are chordoma, chondrosarcoma, and plasmacytoma. Metastatic tumor is probably the most common malignant sacral neoplasm, and the other common systemic tumors are myeloma, Ewing sarcoma, and lymphoma. ${ }^{5}$

Tumors requiring en bloc resection in a "no touch" fashion are metastatic and GCT, chordoma, chondrosarcoma, and Ewing sarcoma. ${ }^{5}$ When these lesions involve the sacrum, partial or total sacrectomy is needed.

Lumbosacral iliac stability depends on the posterior bone and ligaments of the ilium and sacrum. ${ }^{3}$ Sacroiliac stability is not greatly affected by resection if $50 \%$ or more of the sacroiliac joint is intact. ${ }^{13}$ Total sacrectomy, with or without excision of adjacent parts of the ilium, however,

Abbreviations used in this paper: CIA = common iliac artery; $\mathrm{GCT}=$ giant cell tumor. produces enormous osseous and soft-tissue defects causing both vertical and rotational instabilities. Thus, spinopelvic reconstruction is required to reestablish spinopelvic stability.

The objectives of this report are to illustrate-pictorially, radiologically, and experimentally - the total sacrectomy technique and spinopelvic reconstructive methods in light of the biomechanics of the lumbosacral pelvic junction.

\section{ANATOMY AND BIOMECHANICS OF THE LUMBOSACROPELVIC JUNCTION}

The sacrum articulates with the L-5 vertebral body above (lumbosacral joint), the coccyx below (sacrococcygeal joint), and the ilium laterally (sacroiliac joint). The lumbosacral junction is located anterior to the sacroiliac joint, and because of the forward tilt of the sacrum, its weight is transmitted to the superior surface of the sacrum as a rotatory force, which tends to tilt the sacral promontory forward and the apex backward. The axis of such potential rotation may be considered to pass through the center of the S-2 segment. Several osseous and ligamentous constraints keep this rotatory tendency in check. The interosseous and posterior sacroiliac ligaments are the 
strongest ligaments and function to resist forward tilt of the upper end of the sacrum. The sacrotuberous and -spinous ligaments resist the tendency of the lower end of the sacrum and coccyx to tilt backward. The iliolumbar ligaments check the forward migration of L-5 over the sacrum.

The sacroiliac joint is a synovial structure formed between the articular surfaces of the sacrum and ilium. The sacral articular surface is covered by a thick hyaline cartilage, and a thin fibrocartilage overlies the articular surface of ilium. The articular surface of the sacrum that conjoins with ilium has an ear-shaped articular surface. The sacroiliac joint ends at the level of S-2. Complementary irregularities between the articular surfaces offer mechanical stability to the joint. The interosseous and posterior sacroiliac ligaments are the strongest in this region and function to resist the forward tilt of the upper end of the sacrum.

The weight of the body above the pelvis is transmitted through L-5 to the sacrum and then through sacroiliac joints in both the sitting and standing postures. ${ }^{23}$ The sacroiliac joint plays the major role of a transmitting force during sitting and standing, and it functions as a link between the pelvis and spine. Total sacrectomy involving removal of a major part of the sacroiliac joint and iliac bone disrupts this link. Consequently, instability and lowback pain result. Because the patient will not be able to withstand load during walking, standing, and sitting, the recuperative phase will be forestalled.

Sacroiliac stability is not greatly affected by resection of sacral tumors if the sacroiliac joint is left intact. ${ }^{13,28}$ Because the sacroiliac joint ends at S-2, tumors involving the lower sacrum can be removed without substantially destabilizing the pelvis. The posterior sacroiliac ligament also ends at the sacroiliac joint and would therefore not be affected by resections of lower sacral segments.

Gunterberg, et al., ${ }^{13}$ conducted an experimental cadaveric study to demonstrate the pelvic stability after major amputation of the sacrum. They concluded that it was safe for patients undergoing sacral resection to bear weight provided $50 \%$ or more of the sacroiliac joint remained intact.

\section{VASCULAR ANATOMY OF THE SACRAL REGION}

A firm concept of the sacral vascular anatomy is essential for planning major anterior resections and for preoperative embolization. The aorta usually bifurcates into the CIAs at the caudal aspect of the L-4 vertebral body slightly to the left of midline. The CIAs terminate opposite the lumbosacral articulation by dividing into the external and internal iliac arteries.

The right CIA is slightly longer $(5 \mathrm{~cm})$ than the left $(4$ $\mathrm{cm})$ as the aorta bifurcates a little to the left of the midline. The right CIA is crossed in front by the ureter and the ovarian artery in the female. Sympathetic nerve branches cross the artery as well, descending to the hypogastric plexus, the inferior mesenteric artery termination, the sigmoid colon, and the sigmoid mesocolon. To the right of the right iliac artery are located the inferior vena cava, the termination of the right iliac vein, and the psoas muscle. Along the left border of the right CIA are seated the right common iliac vein, the end of the left common iliac vein, and the superior hypogastric plexus.

The shorter left CIA is crossed in front by the ureter, the ovarian artery in the female, branches of the sympathetic nerve, and termination of the inferior mesenteric artery, the sigmoid colon, and the sigmoid mesocolon. To the left of the left CIA is the psoas muscle. On the right side of the artery are the left common iliac vein, the hypogastric plexus, and the middle sacral artery. ${ }^{22}$

The CIA also gives off median and lateral sacral arteries that descend over the sacrum. Presacral tumors often derive their major vascular supply from these arteries. The internal iliac arteries diverge from the CIAs acutely and dive caudally and dorsally into the pelvis. Their first major branches are the iliolumbar arteries that exit dorsally and then course rostrolaterally, crossing over the lumbosacral trunk. One or more lateral sacral arteries are the next branches of the internal iliac artery. They take a paramedian course on top of the piriformis muscle and in close relation to the anterior sacral foramina. The superior gluteal artery is the next and largest branch of the internal iliac artery. It courses posteriorly to penetrate the upper aspect of the sacral plexus at its exit from the pelvis through the greater sciatic foramen. The inferior gluteal artery intersects the lower sacral plexus in its path out of the greater sciatic foramen between the piriformis and coccygeal muscles. ${ }^{26}$

Venous anatomy generally parallels arterial anatomy but is subject to a high degree of variability. Two fairly consistent departures from the arterial schema are the drainage of the middle sacral vein into the left common iliac vein rather than into the vena cava and the drainage of the iliolumbar veins to the common iliac veins rather than into the internal iliac vein. ${ }^{26}$

\section{SACRAL NEURAL ANATOMY}

The sacral neural plexi are formed from the lumbosacral trunk coursing caudally and laterally over the sacral alae with contributions from the anterior rami of S1-3. This union of lumbar and sacral nerve roots occurs over the sacroiliac joint at the level of the S-2 pelvic foramina. The two most proximal branches of the sacral plexus are the superior and inferior gluteal nerves, both exiting the greater sciatic foramen lateral to the plexus with the sciatic nerve. The most important derivative of the sacral plexus is the sciatic nerve that forms at the level of the S3 foramina. Smaller nerves to the quadratus femoris, gemelli, and obturator internus muscles also emanate from the plexus and exit from the pelvis with the sciatic nerve. Two sensory nerves, the posterior femoral cutaneous nerve and the perforating cutaneous nerve, also leave the plexus at this level and exit the greater sciatic foramen. Medial and caudal to the sacral plexus, branches of the anterior rami of S2-4 join to form the pudendal nerve. The pudendal nerve is unique in exiting the greater sciatic foramen only to reenter the lesser sciatic foramen by passing around the sacrospinous ligament. It innervates the coccygeal and levator muscles of the pelvic floor as well as the external anal sphincter. The often ill-defined coccygeal plexus derives from the S-4 and S-5 nerve roots and the coccygeal roots and subserves perianal sensation. 
Both the sympathetic and parasympathetic nerves have an intimate relationship with the sacrum. The sympathetic chains continue caudally into the pelvis, closely applied to the anterior surface of the sacrum. The sympathetic trunks distribute gray rami communicantes to several of the sacral roots and contribute fine filamentous branches to the superior hypogastric plexus. At the S1-2 level, fibers of the superior hypogastric plexus diverge inferolaterally and cross the sympathetic trunks to form two laterally arrayed inferior hypogastric, or pelvic, plexi that overlie the surface of the obturator internus muscles at S2-4. The parasympathetic contribution to the pelvic plexi is derived from small fibers that originate from the anterior S2-4 nerve roots that comprise the pelvic splanchnic nerves. From both the superior hypogastric and the pelvic plexi, small fibers are distributed distally to the various pelvic organs. The sympathetic system promotes timely transport of spermatozoa from the testes to the seminal vesicles and coordinated antegrade ejaculation out of the urethra. The parasympathetic system is primarily responsible for the vascular reflexes that sustain erectile functions. ${ }^{26}$

\section{OPERATIVE TECHNIQUE OF TOTAL SACRECTOMY}

\section{Anterior Procedure (Intraperitoneal Approach)}

Sequential anterior and posterior approaches involving rectus abdominis pull-through pedicle flap reconstruction are used when performing a high sacral resection. The routine use of this flap in the closure of the huge surgical voids created by high sacrectomy has virtually eliminated problems with prolonged wound drainage and breakdown. The aim of the anterior procedure is, first, to expose the anterior aspect of the tumor and, second, to ligate the main tumor vessels. ${ }^{31}$

The patient is positioned supine with appropriate padding at all contact points. After a making a longitudinal midline incision from 2 to $3 \mathrm{~cm}$ above the umbilicus to the symphysis pubis (Fig. 1 left), the retroperitoneal tumor is reached transperitoneally, although the sacrum can be exposed ventrally by a bilateral extraperitoneal dissection. A trans- or extraperitoneal route is used depending on the extent and type of tumor. After the bowel has been "packed off," the posterior parietal peritoneum is opened unilaterally, the ureters are identified and the iliac vessels dissected bilaterally. If the rectum is to be spared, it is mobilized off the tumor capsule ventrally after incision of the retrorectal peritoneal reflection. The tumor capsule is outlined circumferentially. Internal iliac and middle sacral arteries and veins are ligated along with any tumor vessels, and both external arteries should be preserved. Anterior sacrectomy requires mobilization of the iliac veins medially and laterally during the osteotomies (Fig 1 . right). The peritoneal investment and the periosteum are incised caudally as far as the greater sciatic notch, and the cortex can be scored anteriorly to facilitate subsequent completion of the osteotomy from behind. The sacral foramina are visualized anteriorly, and these serve as landmarks to guide the anterior sacral osteotomy. Lateral dissection of the sacral ala allows identification of the lumbar trunk (L4-5) of the lumbosacral plexus. The sacroiliac

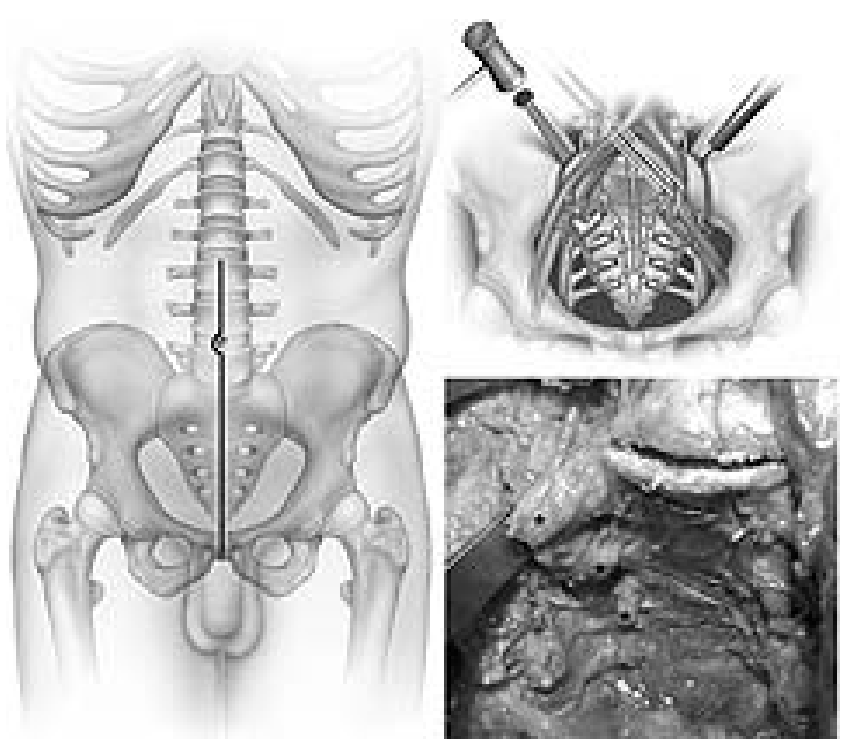

Fig. 1. Left: Drawing showing operative skin incision for anterior sacrectomy. A longitudinal midline incision made from 2 to 3 $\mathrm{cm}$ above the umbilicus to the symphysis pubis. Upper and Lower Right: An anterior sacral osteotomy and L5-S1 discectomy are performed with mobilization of the iliac vessels. The anterior S1-3 nerve roots are divided at their foramina. Waxing the osteotomy cuts helps to reduce bleeding and improves visibility. Asterisks indicate sacral nerve roots $\mathrm{S} 1-3$.

joint is identified lateral to these nerve roots, and a bilateral partial anterior sacroiliac osteotomy is performed using a chisel and/or drill. The L5-S1 disc is exposed and removed along with the anterior aspect of the anulus fibrosus. If the anterior sacral nerve roots are not obscured by the tumor, they are divided at their foramina and cut bilaterally. Waxing the osteotomy cuts helps reduce bleeding and improves visibility. A flexible thin silastic sheath is placed posterior to the vascular structures and the rectum, isolating them from the lumbar vertebrae and sacrum. This inhibits the formation of adhesions between these structures and aids in performing the second stage of the operation. The abdominal skin closure is conducted layer by layer and a drain is placed. After the anterior procedure, a significant week-long episode of ileus can occur because of interruption of the autonomic presacral nerves. ${ }^{11}$

\section{Posterior Approach and Preparation of a Myocutaneous Flap}

A myocutaneous flap preparation is created at the time of the posterior approach. If the posterior approach is continued immediately after the anterior procedure, a myocutaneous flap is made before abdominal closure. At this point, a rectus abdominis myocutaneous flap is prepared based on the inferior epigastric vessels (Fig. 2). The anterior abdominal wall skin island is sealed with iodineimpregnated plastic adhesive dressing, wrapped in an antibiotic-soaked and counted laparotomy pad, and then stowed within the pelvis. It may be helpful for the purpose of hemostasis and for anatomical demarcation during the subsequent posterior approach to use large pieces of gelatin sponge. These pieces are inserted into the plane of 


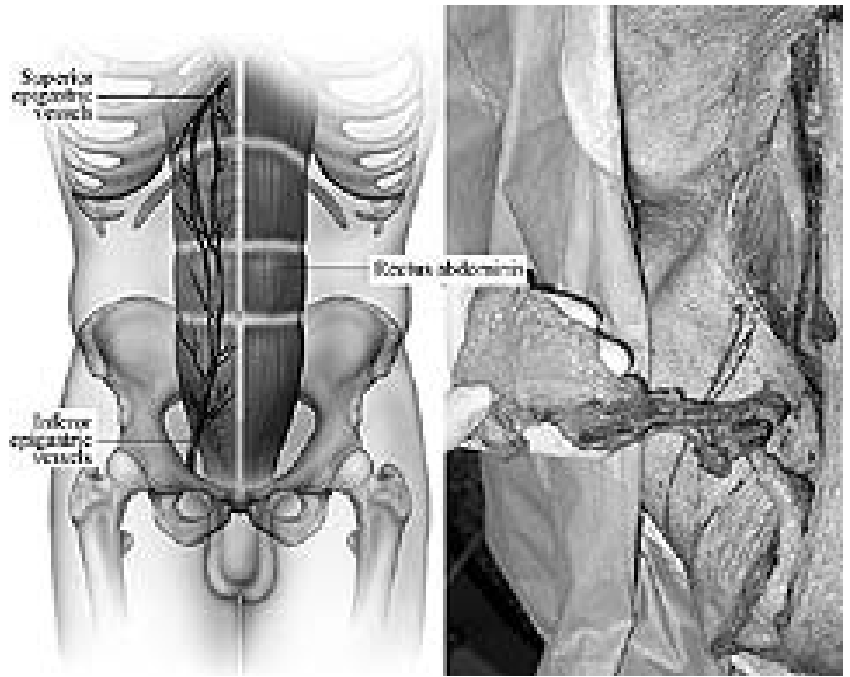

Fig. 2. Left: Drawing showing a rectus abdominis myocutaneous flap based on the inferior epigastric vessels. Right: Photograph showing the rectus abdominis myocutaneous flap. The upper part of the myocutaneous flap has a full layer, which includes the skin and the rectus abdominis muscle containing the inferior hypogastric vessels. The lower portion contains only the rectus abdominis muscle based on the inferior hypogastric vessels.

dissection between the sacrectomy specimen with any attached presacral mass and the iliac vessels, sacral plexi, and the uterus, which are to remain undisturbed. The abdomen is then closed according to the surgeon's preference (Fig. 3). If a colostomy is to be performed, it is usually conducted after the sacrectomy and reconstruction have been completed, to lessen the risks of wound infection.

After the abdominal incision is closed, the patient is placed prone for sacral resection via the posterior midline approach. The posterior incision extends from L-2 to beyond the coccyx, leaving skin, subcutaneous tissue, and

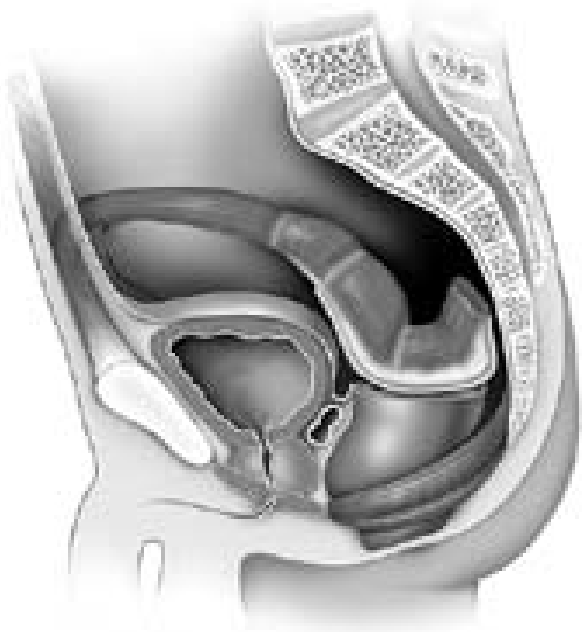

Fig. 3. Sagittal view of the abdomen after abdominal closure; the rectus abdominis myocutaneous flap has already been harvested. The flap is stowed within the pelvic cavity. muscle in place over the sacrum to facilitate en bloc resection. Any skin compromised by tumor, previous biopsy sampling, radiation-induced change, or prior surgery should also be excised with the specimen. A lumbosacral flap is lifted from the sacrum and retracted rostrally. This flap permits wide lateral and caudal exposure without causing ischemic damage and retractor-related muscular injury that may occur with a straight lateral retraction of the posterior lumbosacral musculature (Fig. 4). ${ }^{16}$ Dissection through the soft tissue is deepened in a conical fashion: at the surface of the tissue cone is any tumor- or biopsy specimen-involved skin; within the base is the biopsy tract and any tumor erupting out of the posterior sacrum. Violation of the tumor capsule must be avoided at all costs. The posterior iliac crest, greater sciatic foramina, and sciatic nerves are exposed bilaterally, as are the L3-5 spinous processes, facet joints, and transverse processes. An L-5 and subtotal S-1 laminectomy exposes the thecal sac and cauda equina at this level (Fig. 5). The sacral nerve roots are then divided, and the thecal sac is closed using a double layer of sutures. The remaining disc of L5-S1 is removed completely. The sacrospinalis muscles are sectioned transversely, and the posterior sacroiliac ligament attached to the ilium is incised. Laterally, the gluteal musculature is transected, leaving a cuff attached at its sacral origin. This uncovers the piriformis muscles that are, in turn, divided at their musculotendinous junction. The superior gluteal vessels and nerves are found at the upper border of the pyriformis and the inferior gluteal vessels, and the sciatic, pudendal, and posterior femoral cutaneous nerves are found exiting the pelvis at its lower edge. These should be identified carefully and preserved when-
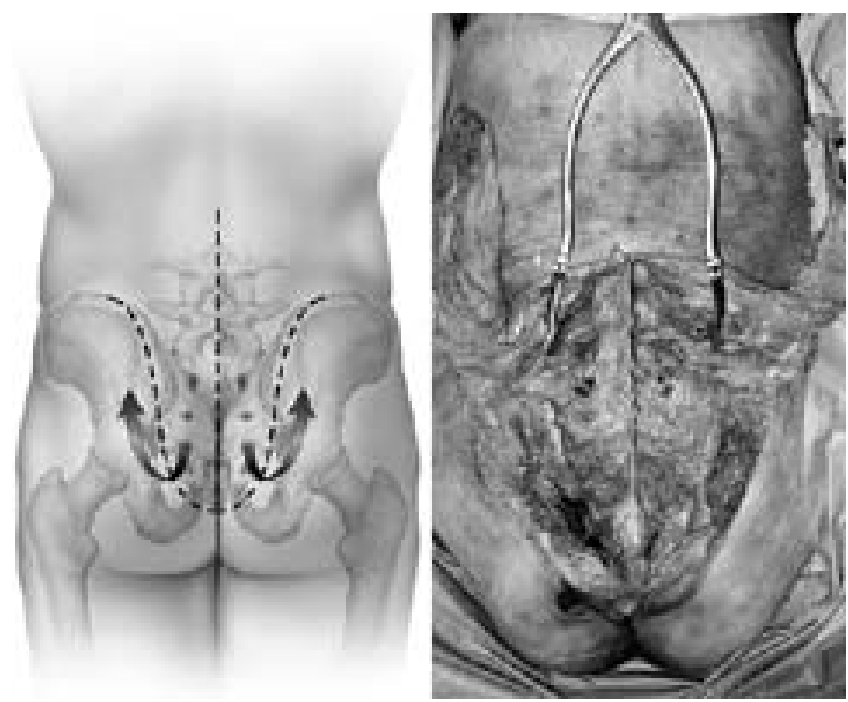

Fig. 4. Skin incision for posterior sacrectomy. Left: Skin incision outlined is used to lift the skin and paraspinal muscles in the sacral area; this permits a wide lateral and caudal exposure without causing ischemic damage and retractor-related muscular injury. The posterior incision extends from L-2 to beyond the coccyx. Right: Exposure for posterior sacrectomy. The posterior iliac crest, medial portion of greater sciatic foramina, as well as the L3-5 spinous processes, facet joints, and transverse processes are identified after skin incision and reflection. 

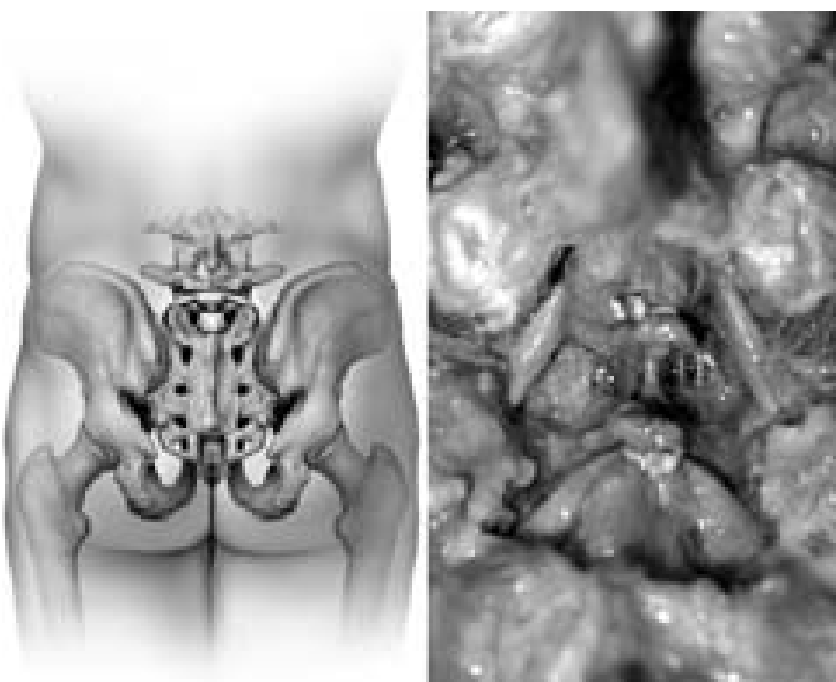

Fig. 5. Drawing (left) and photograph (right) demonstrating L5-S1 laminectomies to expose the thecal sac and cauda equina. Sacral nerve roots are then divided, and the thecal sac is closed using a double layer of sutures. The remaining L5-S1 disc is removed completely.

ever possible. If rectal resection is not planned, the caudal border of the specimen is freed by division of the anococcygeal ligament just proximal to the anal sphincter. The sacrotuberous ligament is detached from the ischial tuberosity, and the coccygeal muscles are cut. The sacrospinous ligament is detached by an osteotomy cut across the base of the ischial spine. If the rectum is to be included with the specimen, the anus is dissected circumferentially and the levator musculature additionally divided. The soft-tissue dissection is finished, and the field is prepared for completion osteotomies from behind (Fig. 6). The previously placed silastic sheath, visualized at this point, serves as a guide in this dissection. The osteotomy along the ilium can enter into the sciatic notch either medial or lateral to the ischial spine. The sacrum is now free and can be lifted out of the wound dorsally. Sacral nerve roots are divided as they exit the sacrum, protecting the sciatic nerves from injury. The entire sacrum along with the neoplasm is then excised en bloc.

\section{Spinopelvic Reconstruction and Closure}

After total sacrectomy (Fig. 7), a spinopelvic reconstruction is required. Various techniques can be used. After completion of the reconstruction, the huge sacrectomy defect must be closed. The rectus abdominis myocutaneous flap, which had been previously positioned in the pelvis, is now retrieved through the sacrectomy window. The flap is trimmed as needed to match the contours of the soft-tissue defect overlying the sacrum and sewn into place in a layered fashion (Fig. 8).

\section{OPERATIVE TECHNIQUES OF SPINOPELVIC RECONSTRUCTION}

After the tumor is resected, and the neural elements are completely decompressed, spinopelvic reconstruction is performed.
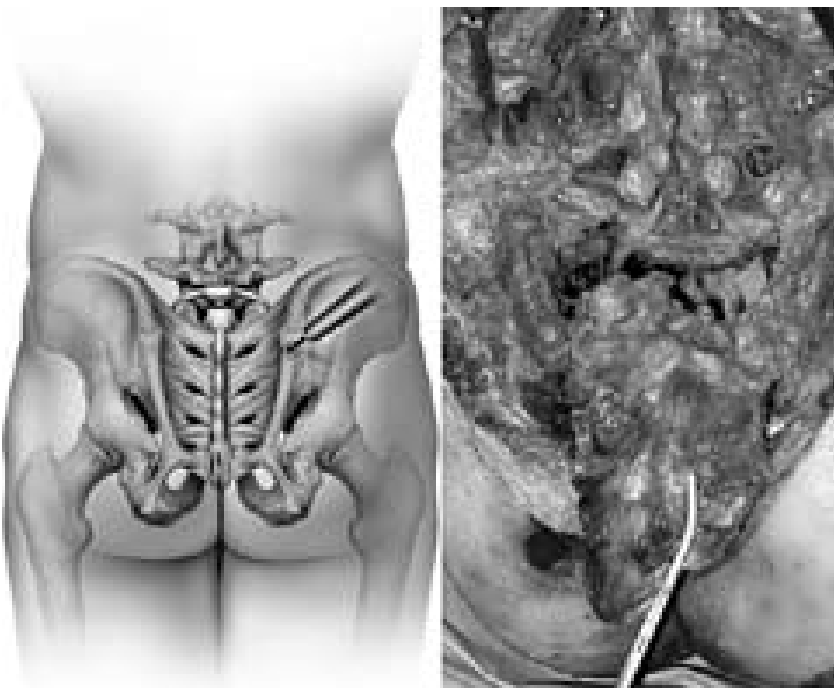

Fig. 6. Technique of posterior sacral osteotomy. Left: Drawing. The sacrospinalis muscles are sectioned transversely, and the posterior sacroiliac ligament is released from the ilium. The sacrotuberous ligament is detached from the ischial tuberosity, and the coccygeal muscles are cut. The osteotomy along the ilium can enter into the sciatic notch either medial or lateral to the ischial spine. Right: Photograph. The sacrum is free and can be lifted out of the wound posteriorly. The entire sacrum and the neoplasm are then removed en bloc.

\section{Modified Galveston Technique}

Initially, pedicle screws (Isola Spinopelvic System; DePuy Acromed, Rayham, MA) are placed bilaterally in the L3-5 lumbar pedicles in the standard fashion. Intraoperative radiography is used to confirm correct positioning. For placement of the Galveston rods in the ilia, a hole is made in the cortex of the medial posterior iliac crest at approximately the S2-3 level. This allows placement of the pilot rod into the cancellous portion of the ilium to create a path for the contoured rod (Isola Spinal Rod Prevent $27.5 \times 95^{\circ}$, DePuy Acromed). This temporary rod is directed to a point $1.5 \mathrm{~cm}$ above the sciatic notch and between the two cortices of the ilium, and it is tapped into place by using a mallet to a depth of $2 \mathrm{~cm}$ or more. This pilot rod is then removed, and a malleable template rod (wire) is inserted, subsequently contoured, and removed. The contoured rod is cut for proper length and bent properly to match the template rod (wire) and is subsequently tapped to a depth of about 4 to $5 \mathrm{~cm}$ into the ilium. The rod is attached to the lumbar pedicle screws. Cross-links are then placed between the rods (Fig. 9). A combination of autograft, allograft, and demineralized bone matrix are used to induce fusion.

\section{Double Iliac Screw Fixation With Posterior Lumbar Fixation}

Intraoperative radiography is used while the lumbar pedicle screws are placed bilaterally in the L3-5 lumbar pedicles in a standard fashion. The iliac screw entry points are identified using a curved osteotome to resect the posterior superior iliac spine. The posterior superior iliac crest should be flush with the sacrum, which places the iliac 


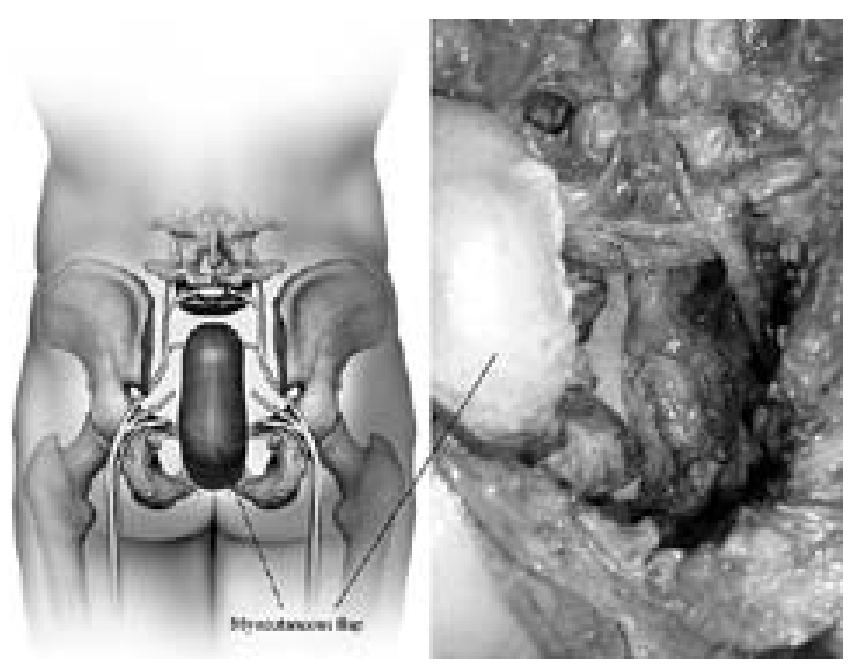

Fig. 7. Drawing (left) and photograph (right) showing the anatomy after completion of total sacrectomy. The pelvic cavity and myocutaneous flap are visualized though the posterior operative field.

foundation more anteriorly and improves the soft-tissue coverage over the ilium. The resection results in an ovalshaped cancellous starting point for both the superior and inferior iliac screws.

With the fingertip of the opposite hand in the superior aspect of the sciatic notch, the iliac starter probe is introduced into the inferior portion of the oval cancellous area and inserted between the two cortical plates of the ilium. The iliac probe is then advanced further between the two cortical plates of the ilium to just above the sciatic notch. A ball tip feeler is inserted into the prepared channel to confirm that the path remains between the iliac cortices

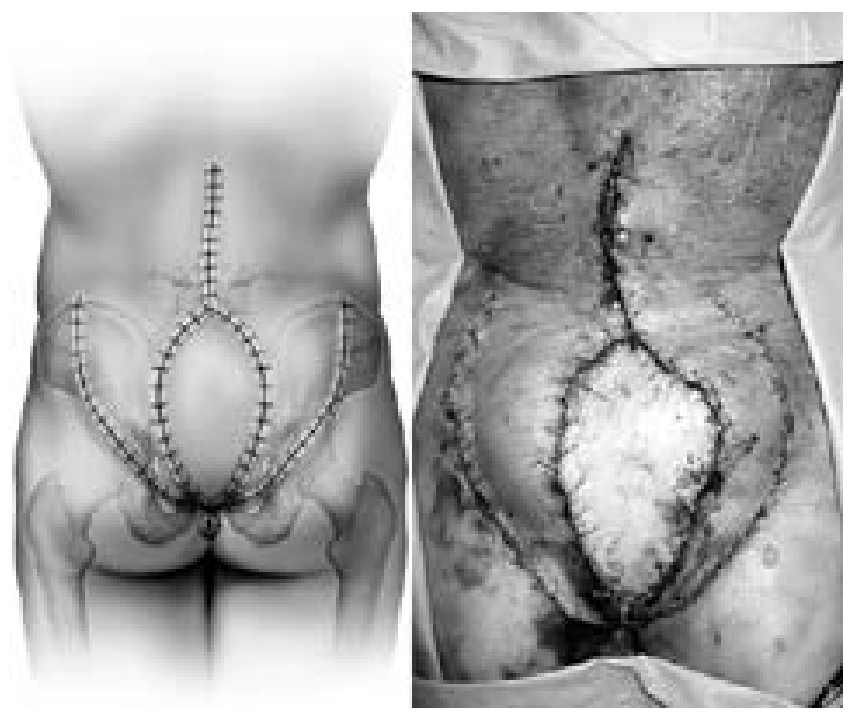

Fig. 8. Drawing (left) and photograph (right) demonstrating the operative incision after completion of spinopelvic reconstruction. The flap is trimmed as needed to match the contours of the soft tissue defect overlying the sacrum and sewn into place in a layered fashion. and has not perforated into the pelvic cavity. The inferior iliac screw path is tapped, and the screw is inserted into the inferior ilium. The inferior iliac screw must be long enough (70-75 $\mathrm{mm}$ in length) to endure weight loading.

The second iliac screw insertion site is identified at the superior end of the oval cancellous area. An iliac starter probe is inserted into the superior end of the oval cancellous region with a slight cephalad inclination. The iliac probe is then inserted to widen the path in the ilium. The superior iliac screw path is tapped, and iliac screw is inserted into the superior ilium. This iliac screw length is 45 to $50 \mathrm{~mm}$. The second screw is inserted, inclined slightly cephalad.

Four rodded connectors and two Isola short rods are required to interconnect the lumbar and iliac screws as follows: two Isola offset long rods (Closed, Offset; $200 \mathrm{~mm}$ ) are used as the main rods. The lumbar pedicle screws are attached to these main rods. Two offset short rods (Closed, Standard, $40 \mathrm{~mm}$ ) are used to connect the main rods and the superior iliac screws. Two short rods are used to connect the inferior iliac screws and the distal ends of the main rods. Cross-links are then placed between the main rods (Fig. 10). A combination of autograft, allograft, and demineralized bone matrix are used to induce fusion.

\section{Various Sacrectomies}

\section{DISCUSSION}

The tumors that require en bloc resection are the GCT, chordoma, chondrosarcoma, and Ewing sarcoma. Metastatic carcinoma, myeloma, plasmacytoma and some forms of Ewing sarcoma are treated by a combination of surgery and medical therapies. Preoperative computerized tomography or magnetic resonance imaging studies, or both, should be performed well above and below the tumor site to define its margins and to determine the rela- 


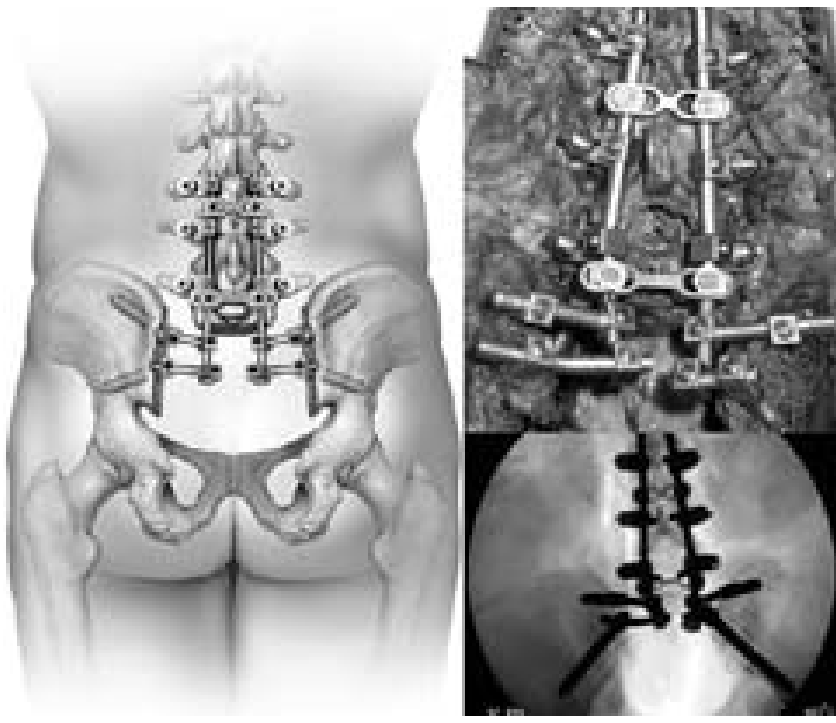

Fig. 10. Left and Upper Right: Drawing and photograph demonstrating completion of the double iliac screw fixation and posterior lumbar segmental fixation. Lower Right: Anteroposterior radiograph revealing the spine after double iliac screw fixation and posterior lumbar segmental fixation.

tionship of the tumor with its surrounding structures. Usually, preoperative arteriography is helpful as well. If the tumor mass extends well beyond the osseous confines and invades surrounding visceral structures, then a preoperative intravenous pyelography or use of a barium enema may be helpful. In cases in which resection of the bladder wall or rectum is planned, diverting colostomies or diverting urinary procedures may be necessary. These must planned and considered in advance of the surgical procedure. $^{5}$

When the decision to operate has been made, the particular approach is dictated by the degree of sacral destruction, the amount of intrapelvic disease, the existence of sacroiliac joint involvement, and the surgical goal of palliation compared with cure.

Sacral Laminectomy. Sacral laminectomy lends itself well to pathological entities originating within and largely confined to the sacral canal because of the ease and clarity with which the neural elements and their coverings are exposed. Treatment of neurogenic tumors and cysts (schwannomas, neurofibromas, and ependymomas) is the foremost indication for this approach. The majority of these tumors are benign, and cure is effected by total resection of the tumor and its capsule. Intimate involvement with the still functional sacral roots is the most frequent impediment to total extirpation of such tumors. Sacral laminectomy as the primary exposure is less well suited to lesions originating outside the sacral canal or extending significantly into the pelvis via the neural foramina or by direct sacral invasion. ${ }^{26}$

Posterior Sacrectomy. The posterior sacrectomy is conducted to treat lesions of the sacrum below the level of the sacroiliac joints. ${ }^{26}$ Because it allows management of the presacral extension of tumor and the potential to perform a genuine oncological procedure (including en bloc resection of the tumor with a circumferential margin of unin- volved tissues), it is distinguished from the sacral laminectomy. These advantages are particularly relevant to osseous lesions of the sacrum that are more often malignant and that are more likely to present with a presacral mass. This is generally suitable for tumors whose superior limit can be reached on digital rectal examination. It should not be used in cases of primary rectal involvement. Candidates should undergo preoperative evaluation for signs of systemic tumor dissemination. ${ }^{26}$

Posterior sacrectomy works well for smaller lesions of the midsacrum and distal sacrum not yet requiring resection above the level of the sacroiliac joint. Although amputation is as high as the first sacral segment, this is a less attractive method for higher sacral resections. It is impossible to dissect the soft tissues of the upper presacrum accurately when using this approach, thereby considerably increasing the risk of major vascular injury, inadvertent entry into the rectum, or violation of the tumor capsule during attempts to osteotomize the anterior sacrum and sacroiliac joints from behind.

These difficulties are best addressed by combining the techniques of posterior sacrectomy with an anterior approach for lesions requiring amputation above the level of the sacroiliac joints.

Anterior-Posterior Sacral Resection. A sequential anterior and posterior approach to facilitate high sacral amputation was described by Bowers ${ }^{4}$ in 1948 . Transabdominal exposure was used to gain control of the hypogastric vessels and enable a safer posterior sacrectomy. The anterior approach obviously improves the surgeon's ability to dissect the tumor accurately from the rectum because it can be accomplished under direct vision rather than by blind finger dissection. Furthermore, should the rectum be involved by tumor either as a consequence of direct extension or because of seeding from an injudicious transrectal biopsy procedure, it can now be freed for en bloc resection with the sacral specimen. This is impossible from the posterior approach alone.

Synchronous Abdominosacral Approach. Localio, et al., ${ }^{17}$ were the first team to popularize a synchronous abdominosacral approach for resection of sacral lesions. They favored a lateral decubitus position, thereby enabling simultaneous anterior and posterior exposure without the need for patient repositioning. They suggested that this technique of synchronous abdominosacral resection resulted in less blood loss than sequential resection. Although this technique can indeed be used to expose the sacrum anteriorly and posteriorly simultaneously in the lateral position, it is more difficult to expose both of them well. The lateral position also complicates efforts at softtissue reconstruction or mechanical stabilization often integral to the success of these procedures.

Sequential Anterior and Posterior Approaches With Myocutaneous Flap. A sequential anterior and posterior approach with pedicled rectus abdominis pull-through flap reconstruction is favored in cases requiring high sacral resections. Our resection technique is similar to that described by Stener and Gunterberg. ${ }^{28}$ The rectus abdominis pull-through flap was originally described for reconstruction and repair of perineal wounds. ${ }^{25,30}$ Its routine use in the closure of the huge surgical voids created by high sacrectomy has virtually eliminated problems with 
prolonged wound drainage as well as breakdown and instrument-related pressure necrosis in thin patients. Although the surgery is necessarily interrupted by the need for patient repositioning, its associated superior visibility and the working ease more than justifies this inconvenience. Other methods are the alternatives of primary closure or reconstruction with reversed latissimus dorsi flaps, gluteal flaps, or gracilis flaps. Microvascular free flap reconstruction is yet another option, but this further escalates the technical requirements of an already arduous procedure. The rectus abdominis pull-through flap is recommended especially in the not infrequent circumstances of previous surgery or irradiation, when the prospects for successful primary closure are nil. It also largely averts problems with rectal prolapse into the wound during defecation and obviates the need for mesh closure of the posterior peritoneal or fascial defect. ${ }^{26}$

Decision regarding total or subtotal sacrectomy for en bloc resection can be made after radiological evaluations and bone biopsy. Total sacrectomy implies removal of the osseous sacrum and destruction of the peripheral nerves and autonomic nervous system below the L-5 nerve roots, and thus additional extensive reconstructive surgery is required.

Gokaslan, et al., ${ }^{12}$ performed total sacrectomy and reconstruction in two patients with GCT and reviewed outcomes in cases of total sacrectomy reported in the literature. The blood loss associated with a total sacrectomy can be high, ranging from 6.5 to $40 \mathrm{~L}$ for single-stage operations to 10.1 to $80 \mathrm{~L}$ for two-stage procedures. Two patients were able to walk unassisted by a cane and three were able to walk with canes and no braces postsurgery.

Tomita and Tsuchiya ${ }^{31}$ reported on three cases (two GCTs and one chordoma) in which total sacrectomy and reconstruction were performed. There were no recurrences. Massive bleeding was a problem. The amount of total bleeding was approximately $11,000 \mathrm{ml}$ in their cases. Neurological deficit was another problem. Sphincter disturbance of bladder and rectum occurs after amputation below the S-1 nerve roots; however, control by self-catheterization and enema was acceptable, and thus cystostomy and colostomy were not needed. Regarding lowerextremity function, if L-5 nerve roots were preserved, a patient could walk using a short leg brace or without orthosis, when muscle strength of ankle joint flexion was Grade 3 to 4 in their series. Gokaslan, et al., ${ }^{12}$ considered radical resection to be the best treatment for primary malignant tumors involving the entire sacrum. This was especially true for aggressive sacral neoplasms such as GCTs that have a low tendency to metastasize but have a high recurrence rate after conservative resections.

We think total sacrectomy via an anterior-posterior approach is the only oncologically reasonable way to excise a huge sacral tumor en bloc. Although it is aggressive surgery, which destroys the nervous system below the L-5 nerve roots, it is indicated in selected patients.

\section{SPINOPELVIC RECONSTRUCTIONS AFTER TOTAL SACRECTOMY}

\section{Indications of Spinopelvic Reconstruction}

Structurally adequate sacral integrity is also achieved with the retention of the sacral ilia and the sacral body above the S-1 neural foramina. This preserves enough of the sacral ala and the lumbopelvic ligaments to provide adequate stability. ${ }^{3}$ Localio, et al., ${ }^{17}$ reported the absence of instability after resection at the S1-2 interspace. Gennari, et al., ${ }^{11}$ described a series of eight patients with sacrococcygeal chordoma in whom high sacral resection through S-2 was performed via a posterior approach; they found stability to be present after resection. Hays ${ }^{15}$ reported the resection of a benign GCT. The procedure involved excision of the posteroinferior portion of the sacral joint bilaterally without stabilization. There were no cases of instability found in the two-year follow-up period.

In some reports reconstruction was not conducted after total sacrectomies; instead, patients were maintained in hip spicas for prolonged periods, with ambulation dependent on scar tissue. .,33 $^{8,33}$

Based on the excellent study by Gunterberg, et al., ${ }^{13}$ absolute indications for spinopelvic stabilizations are 1) total sacrectomy in which the whole sacroiliac joint is removed and 2) partial sacrectomy involving more than $50 \%$ of sacroiliac joint each side.

\section{Spinopelvic Reconstruction Techniques}

The various spinopelvic reconstruction techniques are reported in the literature. These include sacroiliac joint screw fixation, ${ }^{14}$ iliac-sacral screw fixation,, 20 posterior iliosacral plating and screw fixation, ${ }^{10,20}$ custom-made prosthesis, ${ }^{32,33}$ Galveston rod fixation, ${ }^{1,6,7,12,26}$ iliac screws, and transiliac rods.

In the treatment of sacral fracture and sacroiliac disruption, the sacroiliac joint screw technique ${ }^{14}$ has evolved. We believe that this technique is suited for a partial sacrectomy in which nearly $50 \%$ of the sacroiliac joint remains. It would be more difficult to achieve adequate screw purchase if the joint is affected by severe osteoporosis.

The iliac-sacral screw fixation technique ${ }^{9,20}$ via the posterior approach is recommended after partial removal of the sacrum. This technique has been shown to be associated with significantly less failure than the Galveston technique. It can be used in conjunction with hooks or pedicle screws in the lumbar spine above. ${ }^{9}$

The posterior iliosacral plating and screw fixation technique $^{10,20}$ via a posterior approach after partial removal of the sacrum requires the same surgical route and exposure as placement of transiliac bars. This technique is often used in conjunction with sacroiliac joint screws. One potential complication is the pressure necrosis over the skin in a very thin patient.

In another interesting report, Salehi, et al., ${ }^{24}$ presented a novel reconstructive technique for spinopelvic stabilization after resection of metastatic sacral tumors in three cases involving a modification of the transiliac bar. They used a cage and the ilial bolt system for fixation of spine to the ilium and connected the rods that had been affixed to the pedicle screws with satisfactory results.

A custom-made prosthesis ${ }^{32,33}$ is an alternative method of reconstruction after sacrectomy. Based on review of the literature, this technique has been applied only in case reports. The disadvantages of this technique are the inability to make adjustments intraoperatively and the cost of the prosthesis.

Galveston Rod Technique and Modification. The Galveston rod technique was first described for the treatment 
of neuromuscular scoliosis with pelvic obliquity. ${ }^{1,2}$ This fixation is suitable for reconstruction in a total sacrectomy, which requires bypass stabilization of ilium to the lumbar spine. The modified Galveston rod technique has been reported by many authors. $1,6,7,9,12,19,26$

Originally, the rod was use in conjunction with sublaminar wiring of the lumbar spine. These constructs, however, do not provide substantial torsional stability or resistance to extension. ${ }^{21}$ At present, pedicle screws provide sig nificantly increased rigidity compared with sublaminar devices. When attached to the rods and cross-linked the medially directed screw creates a triangular effect, which greatly increases the screw pullout resistance, increases torsional stability, and allows a short-segment fusion. ${ }^{6,719,27}$ A potential drawback is the relative difficulty of rod contouring. Preformed rods (Isola iliac screw; Acromed) can be obtained, which can decrease intraoperative time.

Jackson and Gokaslan ${ }^{16}$ retrospectively reviewed cases of spinopelvic reconstruction in which a modification of the Galveston technique had been used in 13 patients with lumbosacral neoplasms. Ambulatory status improved in eight patients. They reported significant relief of spinal pain, which was measured by the visual analog scale as well as medication consumption. They concluded that Galveston L-rod pelvic fixation is an effective means of achieving stabilization that can provide significant pain relief and preserve ambulatory capacity.

McCord, et al. ${ }^{19}$ discussed the lumbosacral pivot point, which is the axis of rotation (flexion and extension) in the lumbosacral region. It is located at the level of the posterior anulus fibrosus of the L5-S1 motion segment. Posterior implants providing a moment arm that extends anterior to this point can effectively resist rotation. An implant that is extended caudal to this point can also provide a biomechanical advantage.

In the case of total sacrectomy, however, there is no bone for sacral fixation. Bypass fixation or iliac fixation is the only procedure to stabilize the spine-pelvis junction. For a biomechanical advantage, the screw or rod that projects through the iliac bone should be anterior to the lumbosacral pivot point. This is necessary to achieve adequate moment arm length to prevent pelvic flexion. ${ }^{3}$

The Galveston rod technique and its modifications, in which the iliac rod extends anterior to the lumbosacral pivot point, provide a long lever arm within the ilium to counteract the forces exerted by the lumbar spine. This fixation technique appears to provide a strong lumbosacropelvic fixation. ${ }^{19}$

Double Iliac Screw Fixation With Posterior Lumbar Fixation. Margulies, et al. ${ }^{18}$ reported that the placement of long 7- to 8-mm-diameter threaded screws into the iliac wing could be a technically easier method for iliolumbar fixation than the Galveston technique. After placement of screws in optimal position, they are linked to the spinal rod independently. A cross-linked bar between the rods makes the construct more rigid and stable. Sung, et al., ${ }^{29}$ reported the successful results of combining structural allograft and screw fixation through the ilium to bridge the osseous defect after subtotal or total removal of sacrum in two patients.

We used the modified Galveston technique because it is the basic procedure for the reconstruction of the spinopelvic area. Our preferred fixation method involves two iliac screws in each ilium, which connect the rods from the lumbar spine. This is biomechanically stronger because it counteracts the rotational force of the spinopelvic axis more tightly than the modified Galveston technique.

\section{CONCLUSIONS}

In cases in which tumor extirpation requires total sacrectomy, the procedure is demanding for the surgeon unfamiliar with anterior sacral anatomy and who must reestablish spinopelvic stability. The collaborative input of oncological and plastic surgeons may be of major assistance to spine surgeons who undertake a high sacral resection. We believe that this assistance should benefit the patients in terms of enhanced outcomes. There are several methods by which to satisfy the imperative for a tensionless wound closure with obliteration of dead space by viable nonirradiated tissues. The rectus abdominis pullthrough flap has been of major strategic benefit.

Various methods of stabilization and instrumentation have been reported in the literature. We have used the modified Galveston technique, double iliac screw fixation, and posterior lumbar segmental fixation. Understanding the nature of the disease as well as the biomechanics of the lumbosacropelvic area and fixation will help surgeons to select the appropriate fixation technique.

\section{References}

1. Allen BL Jr, Ferguson RL: The Galveston technique for L rod instrumentation of the scoliotic spine. Spine 7:276-284, 1982

2. Allen Br Jr, Ferguson RL: The Galveston technique of pelvic fixation with L-rod instrumentation of the spine. Spine 9: 388-394, 1984

3. Benzel EC: Biomechanics of Spine Stabilization. Rolling Meadows, IL: AANS Press, 2001, pp 297-309

4. Bowers RF: Giant cell tumor of the sacrum: a case report. Ann Surg 128:1164-1172, 1948

5. Bridwell KH: Management of tumors at the lumbosacral junction, in Margulies JY, Floman Y, Farcy JPC, et al (eds): Lumbosacral and Spinopelvic Fixation. Philadelphia: LippincottRaven, 1996, pp 109-122

6. Carlson GD, Abitbol JJ, Anderson DR, et al: Screw fixation in the human sacrum. An in vitro study of the biomechanics of fixation. Spine 17 (Suppl 6):196-203, 1990

7. Carson WL, Duffield RC, Arendt M, et al: Internal forces and moments in transpedicular spine instrumentation: the effect of pedicle screw angle and transfixation-the 4R-4bar linkage concept. Spine 15:893-901, 1990

8. Edwards CC: Spinal reconstruction in tumor management, in Uhthoff HK (ed): Current Concepts of Diagnosis and Treatment of Bone and Soft Tissue Tumors. Berlin: SpringerVerlag, 1984, pp 329-349

9. Freeman BL III: Scoliosis and kyphosis in Canale ST (ed): Campbell's Operative Orthopedics, ed 9. St. Louis: Mosby, 1998, Vol 3, pp 2918-2923

10. Fuchs B, Yaszemski MJ, Sim FH: Combined posterior pelvis and lumbar spine resection for sarcoma. Clin Orthop 397: $12-18,2002$

11. Gennari L, Azzarelli A, Quagliuolo V: A posterior approach for the excision of sacral chordoma. J Bone Joint Surg Br 69: 565-568, 1987

12. Gokaslan ZL, Romsdahl MM, Kroll SS, et al: Total sacrectomy 
and Galveston L-rod reconstruction for malignant neoplasm. Technical note. J Neurosurg 87:781-787, 1997

13. Gunterberg B, Romanus B, Sterner B: Pelvic strength after major amputation of the sacrum. An experimental study. Acta Orthop Scand 47:635-642, 1976

14. Guyton JL: Fractures of hip, acetabulum, and pelvis, in Canale ST (ed): Campbell's Operative Orthopedics, ed 9. St. Louis: Mosby, 1998, Vol 3 p 2262

15. Hays RP: Resection of the sacrum for benign giant cell tumor: a case report. Ann Surg 138:115-120, 1953

16. Jackson RJ, Gokaslan ZL: Spinal-pelvic fixation in patients with lumbosacral neoplasms. J Neurosurg (Spine 1) 92: 61-70, 2000

17. Localio SA, Eng K, Ranson JH: Abdominosacral approach for retrorectal tumors. Ann Surg 191:555-560, 1980

18. Margulies JY, Armour EF, Kohler-Ekstrand C, et al: Revision of fusion from the spine to the sacropelvis: considerations, in Margulies JY, Aebi M, Farcy JPC (eds): Revision Spine Surgery. St. Louis: Mosby, 1999, pp 623-630

19. McCord DH, Cunningham BW, Shono Y, et al: Biomechanical analysis of lumbosacral fixation. Spine 17 (Suppl 8):235-243, 1992

20. Norris BL, Bosse MJ, Kellam JF, et al: Pelvic fractures: sacral fixation, in Wiss DA (ed): Fractures. Philadelphia: LippincottRaven, 1998, pp 613-629

21. Ogilvie JW, Bradford DS: Sublaminar fixation in lumbosacral fusions. Clin Orthop 269:157-161, 1991

22. Pait TG, Elias AJR, Tribell R: Thoracic, Lumbar, and Sacral Spine Anatomy for Endoscopic Surgery. Neurosurgery 51 (Suppl 5):67-78, 2002

23. Rengachary SS: Surgical anatomy of the sacrum, in Doty JR, Rengachary SS (eds): Surgical Disorders of the Sacrum. New York: Thieme, 1994, pp 21-33

24. Salehi SA, McCafferty RR, Karahalios D, et al: Neural function preservation and early mobilization after resection of the metastatic sacral tumors and lumbosacropelvic junction reconstruc- tion: Report of three cases. J Neurosurg (Spine 1) 97:88-93, 2002

25. Skene AI, Gault DT, Woodhouse CR, et al: Perineal, vulval and vaginoperitoneal reconstruction using the rectus abdominis myocutaneous flap. Br J Surg 77:635-637, 1990

26. Smith DA, Kumar R, Cahill DW: Sacral lesions, in Benzel EC (ed): Spine Surgery: Techniques, Complication, Avoidance, and Management. Philadelphia: Churchill Livingstone, 1999, pp741-758

27. Smith SA, Abitbol JJ, Carlson GD, et al: The effects of depth of penetration, screw orientation, and bone density on sacral screw fixation. Spine 18:1006-1010, 1993

28. Stener B, Gunterberg B: High amputation of the sacrum for extirpation of tumors. Principles and techniques. Spine 3: 351-366, 1978

29. Sung HW, Shu WP, Wang HM, et al: Surgical treatment of primary tumors of the sacrum. Clin Orthop 215:91-98, 1987

30. Tobin GR, Day TG: Vaginal and pelvic reconstruction with distally based rectus abdominis myocutaneous flaps. Plast Reconstr Surg 81:62-73, 1988

31. Tomita K, Tsuchiya H: Total sacrectomy and reconstruction for huge sacral tumors. Spine 15:1223-1227, 1990

32. Wuisman P, Lieshout O, van Dijk M: Reconstruction after total en bloc sacrectomy for osteosarcoma using a custom-made prosthesis: a technical note. Spine 26:431-439, 2001

33. Wuisman $P$, Lieshout O, Sugihara S, et al: Total sacrectomy and reconstruction: oncologic and future outcome. Clin Orthop 381:192-203, 2000

Manuscript received June 19, 2003.

Accepted in final form July 10, 2003.

Address reprint requests to: Daniel H. Kim, M.D., Department of Neurosurgery, Stanford University Medical Center, 300 Pasteur Drive, Edward Building Room R-201, Stanford, California 943055327. email: neurokim@ stanford.edu. 